\title{
Prediction of hepatitis C virus interferon/ribavirin therapy outcome based on viral nucleotide attributes using machine learning algorithms
}

\author{
Amir Hossein KayvanJoo ${ }^{1}$, Mansour Ebrahimi ${ }^{1 *}$ and Gholamreza Haqshenas ${ }^{1,2^{*}+}$
}

\begin{abstract}
Background: Hepatitis C virus (HCV) causes chronic hepatitis C in 2-3\% of world population and remains one of the health threatening human viruses, worldwide. In the absence of an effective vaccine, therapeutic approach is the only option to combat hepatitis C. Interferon-alpha (IFN-alpha) and ribavirin (RBV) combination alone or in combination with recently introduced new direct-acting antivirals (DAA) is used to treat patients infected with HCV. The present study utilized feature selection methods (Gini Index, Chi Squared and machine learning algorithms) and other bioinformatics tools to identify genetic determinants of therapy outcome within the entire HCV nucleotide sequence.

Results: Using combination of several algorithms, the present study performed a comprehensive bioinformatics analysis and identified several nucleotide attributes within the full-length nucleotide sequences of HCV subtypes $1 \mathrm{a}$ and $1 \mathrm{~b}$ that correlated with treatment outcome. Feature selection algorithms identified several nucleotide features (e.g. count of hydrogen and CG). Combination of algorithms utilized the selected nucleotide attributes and predicted HCV subtypes $1 \mathrm{a}$ and $1 \mathrm{~b}$ therapy responders from non-responders with an accuracy of $75.00 \%$ and $85.00 \%$, respectively. In addition, therapy responders and relapsers were categorized with an accuracy of $82.50 \%$ and $84.17 \%$, respectively. Based on the identified attributes, decision trees were induced to differentiate different therapy response groups.

Conclusions: The present study identified new genetic markers that potentially impact the outcome of hepatitis C treatment. In addition, the results suggest new viral genomic attributes that might influence the outcome of IFN-mediated immune response to HCV infection.
\end{abstract}

\section{Background}

Hepatitis $\mathrm{C}$ virus (HCV) is a blood-borne virus, which causes chronic hepatitis in humans. Despite its discovery over 2 decades ago [1], HCV remains one of the major health threatening infectious agents worldwide. Recent estimations indicate that approximately $2-3 \%$ of world population (125-175 million) suffer from chronic hepatitis C [2]. So far, at least six major HCV genotypes (1-6) with less than $72 \%$ nucleotide identities, each comprised of several subtypes $(1 \mathrm{a}, 1 \mathrm{~b}$, etc.) with $75-86 \%$ nucleotide identities, have been identified. The single-stranded viral RNA genome with a size of $\sim 9.6 \mathrm{~kb}$ replicates through a

\footnotetext{
* Correspondence: Mansour@future.org; Haqshenas@yahoo.com

${ }^{\dagger}$ Equal contributors

'Department of Biology, School of Basic Sciences, University of Qom, Qom, Iran 2Present address: Microbiology Department, Monash University, Melbourne, Australia
}

\section{Ciomed Central}

(c) 2014 KayvanJoo et al.; licensee BioMed Central Ltd. This is an Open Access article distributed under the terms of the Creative Commons Attribution License (http://creativecommons.org/licenses/by/4.0), which permits unrestricted use, distribution, and reproduction in any medium, provided the original work is properly credited. The Creative Commons Public Domain Dedication waiver (http://creativecommons.org/publicdomain/zero/1.0/) applies to the data made available in this article, unless otherwise stated. double-stranded intermediate form. High frequency of point mutations in the $\mathrm{HCV}$ genome during virus replication and the virion structure [3] are major factors hindering the development of a preventive vaccine. To identify an effective therapeutic approach, HCV biology and viral structural (core, E1, and E2) and non-structural (NS) (NS2-3, NS4A-B, NS5A-B) proteins have been extensively studied. Currently, therapeutic regimens for treatment of $\mathrm{HCV}$-infected patients involve HCV direct-/indirectacting antivirals. Combination of pegylated interferonalpha (IFN-alpha) and ribavirin (RBV) is prescribed by physicians for treatment of hepatitis C. IFN, a known broadly acting antiviral cytokine, is an essential component of innate immune response. The exact mechanism of action of RBV remains unknown although it improves 
response rate when it is combined with interferon [4]. The recently FDA approved direct-acting antivirals (DAA, telaprevir and boceprevir) that are used in combination with IFN/RBV have improved HCV therapy success rate by 16$40 \%[5,6]$. Long-term IFN/RBV combination treatment (24-48 weeks) is required to achieve sustained virological response (SVR). Some patients resolve the virus at the completion of treatment (responders), of whom a proportion demonstrate a virus rebound within 6 months posttreatment (relapsers). Some HCV patients are resistant to combination therapy (non-responders). The success rate of HCV treatment depends on many host and viral factors. Patients who are chronically infected with HCV genotype 1 poorly respond to the combination treatment (about $50 \%$ SVR) while higher response rate is observed when patients are infected with genotypes 2 and 3 (about 70-80\% SVR). The genotype-dependent therapy response rate suggests that the composition of viral nucleotide and amino acid sequences may impact the therapy outcome. Several comparative analyses have indicated that the amino acid sequences of the $\mathrm{HCV}$ proteins including core $[7,8], \mathrm{E} 2$ [9-13], p7 [13], NS2 [13,14], NS5A [10-13,15] and NS5B [16] may the success rate of the combination treatment. In addition, host parameters including genetic polymorphism in IL28B locus have been indicated as therapy response rate determinants $[17,18]$.

There is insufficient data describing nucleotide attributes that correlate with response to therapy. In addition, genomic determinants that may predict the relapse of the disease following a successful clearance remain unclear. This study aims to use various clustering, screening, and decision tree models to analyse full-length HCV genomes and identify novel genetic markers for the prediction of HCV therapy outcome.

\section{Results}

The initial dataset contained 93 full-length nucleotide sequences of HCV subtypes 1a and 1b from Virahep study [19]. A summary of all data processing steps adopted in this study to predict therapy outcome has been presented in Figure 1. For each sequence, 76 gene attributes were computed. Using data filtering algorithms, useless and closely related attributes (correlations higher than 95\%) were excluded. Overall, 35-37 attributes were identified useful for the identification of different treatment outcome groups.

\section{Attribute weighting}

The importance and the contribution of each useful attribute in building the target variable (response to treatment) was evaluated by attribute weighting algorithms. Data was normalized to give a value between 0 and 1 to each weight [20-23]. The important attributes and the number of models which allocated a weight above 0.5 to each attribute have been presented in Table $1 \mathrm{~A}$ and $\mathrm{B}$, respectively. All Attributes and the relevant weighting models have been presented in an Additional file (see Additional file 1). Only attributes that gained weights higher than 0.5 were used by prediction and tree induction algorithms to predict the response to treatment.

\section{Trees induction}

A decision tree is constructed by looking for regularities in data, determining the features to add at the next level of the tree using an entropy calculation, and then choosing the feature that minimizes the entropy impurity [24]. As shown in Figure 2, a tree model for subtype 1a responders vs. non-responders was generated based on the double strand count of nitrogen and hydrogen. A nitrogen value $\leq 68358.50$ classified some sequences as responders though it did not distinguish all responders. When a nitrogen value is $\geq 68358.50$, therapy outcome depends on the hydrogen count value. Figure 3 is a tree model built on the analysis of subtype 1a responders vs. relapsers' sequences detected the oxygen count as the root of the tree and sequences with a value $>63177$ were identified as responder group. When the oxygen value was $<63177$, the therapy outcome depends on the count of UU. Figures 4 and 5 represent the tree models developed for subtype $1 \mathrm{~b}$ responders vs. non-responders and responders vs. relapsers' sequences, respectively.

\section{Prediction algorithms}

Various machine based learning algorithms [decision tree, Support Vector Machine (SVM), Naive Bayes and Neural Networks] were trained and tested to predict IFN/RBV therapy respond based on computed features. When an algorithm gained the highest accuracy it was used as fundamental predictor for IFN/RBV response treatments.

\section{Decision tree}

Decision tree classifiers are the most popular supervised learning methods for data exploration. The trees summarize and transform data into a more compact forms that maintain the essential characteristics for an easy interpretation [25].

The best performance among 176 decision tree models (16 models run on 11 datasets) in the prediction of therapy response are presented in Table 2A and B. The highest accuracy values for the prediction of subtypes $1 \mathrm{a}$ and 1b responders from non-responders were $69.17 \%$ and $80.00 \%$, respectively. The highest accuracy $(81.67 \%)$ was achieved for both subtypes when responder and relapser groups were analysed.

\section{SVM approach}

SVM algorithms have become very popular as a high-performance classifier in several fields including 


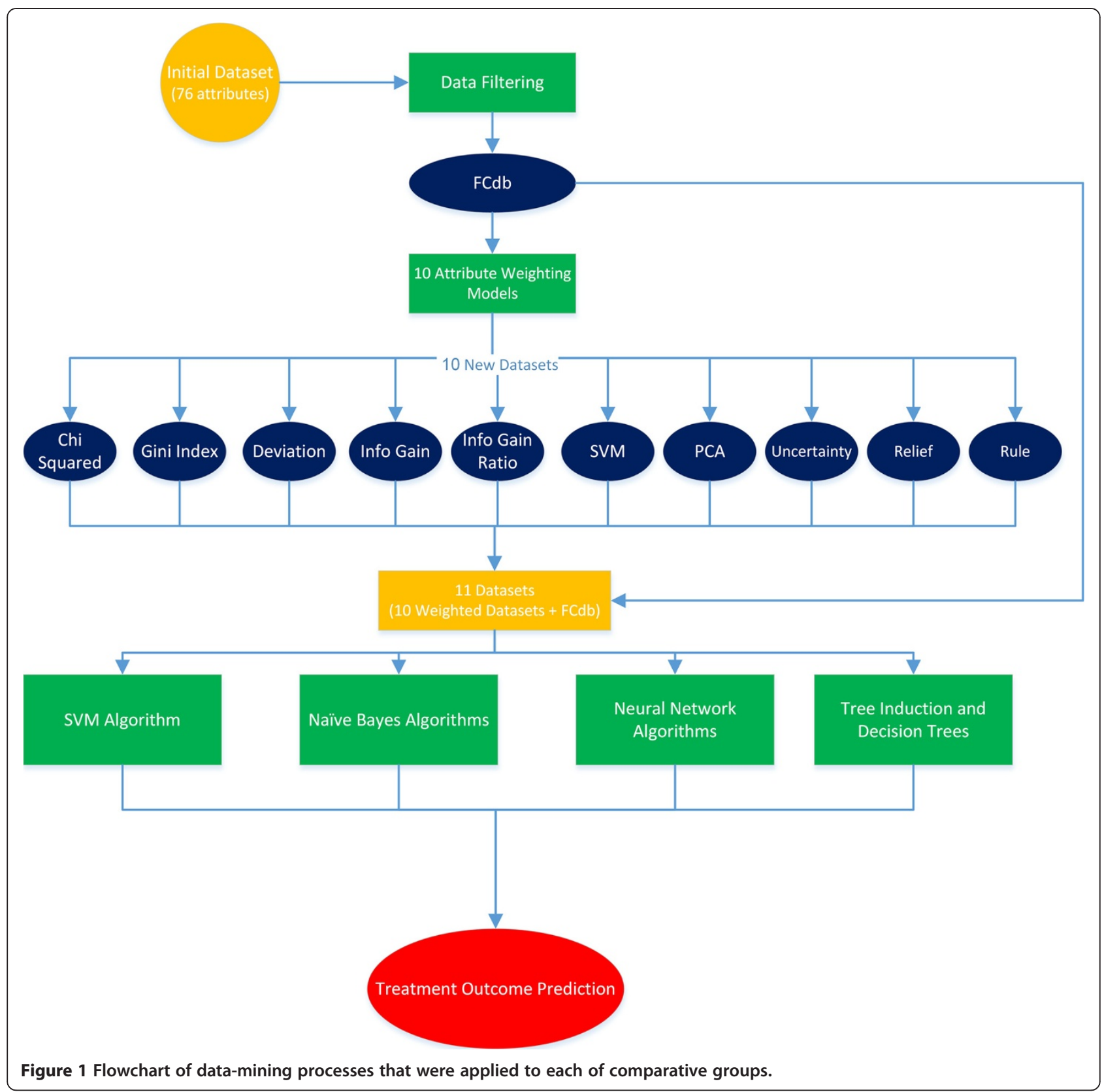

bioinformatics. The main objective is to construct a hyperplane as the decision surface so that the margin of separation between positive and negative examples is maximized [25].

The best accuracy values for the $1 \mathrm{a}$ and $1 \mathrm{~b}$ responder vs. non-responder sequences using SVM analysis showed $74.17 \%$ and $75.00 \%$ accuracies, respectively. SVM analysis revealed higher accuracies up to $82.50 \%$ and $84.17 \%$ for responder vs. relapser of subtypes $1 \mathrm{a}$ and $1 \mathrm{~b}$ sequences, respectively. A summary of results including sensitivity, specificity, precision, recall and F-measure have been provided in Additional files 2 and 3.

For SVM analysis on the $1 \mathrm{a}$ and $1 \mathrm{~b}$ responder vs. nonresponder sequences, the best accuracy values were
$74.17 \%$ and $75.00 \%$, respectively. The accuracies of our approaches were estimated to be higher when responder vs. relapser of subtype 1a sequences $(82.50 \%)$ and responder vs. relapser of subtype $1 \mathrm{~b}$ sequences $(84.17 \%)$ were analyzed. Accuracy values and other parameters (sensitivity, specificity, precision, recall and F-measure) have been presented in Additional files 2 and 3.

\section{Naïve Bayes}

The Naive Bayes classifier is a simple learning algorithm that is used for data mining applications. This algorithm can also be used as a predictive model. 
Table 1 Most important nucleotide attributes that were selected by different weighting algorithms

\begin{tabular}{|c|c|c|c|}
\hline \multicolumn{4}{|l|}{$A$} \\
\hline \multicolumn{2}{|c|}{$\begin{array}{c}\text { Subtype 1a (Responders vs. } \\
\text { Non-Responders) }\end{array}$} & \multicolumn{2}{|c|}{$\begin{array}{c}\text { Subtype } 1 \mathrm{~b} \text { (Responders vs. } \\
\text { Non-Responders) }\end{array}$} \\
\hline Attribute & $\begin{array}{l}\text { No. of selective } \\
\text { attribute } \\
\text { weightings } \\
\text { (out of 10) }\end{array}$ & Attribute & $\begin{array}{c}\text { No. of selective } \\
\text { attribute } \\
\text { weightings } \\
\text { (out of 10) }\end{array}$ \\
\hline Count of hydrogen & 9 & Count of GC & 8 \\
\hline Count of oxygen & 8 & Count of UA & 7 \\
\hline Count of CA & 7 & $\begin{array}{l}\text { DS Count of } \\
\text { nitrogen }\end{array}$ & 7 \\
\hline Count of CG & 7 & Count of $\mathrm{AU}$ & 6 \\
\hline Count of Cytosine & 7 & Count of GG & 5 \\
\hline Count of Guanine & 7 & Count of Uracil & 5 \\
\hline Count of GU & 6 & & \\
\hline Count of UU & 5 & & \\
\hline Count of UA & 5 & & \\
\hline Count of CC & 5 & & \\
\hline \multicolumn{4}{|l|}{ B } \\
\hline \multicolumn{2}{|c|}{$\begin{array}{c}\text { Subtype } 1 \text { a (Responders vs. } \\
\text { Relapsers) }\end{array}$} & \multicolumn{2}{|c|}{$\begin{array}{c}\text { Subtype } 1 \mathrm{~b} \text { (Responders vs. } \\
\text { Relapsers) }\end{array}$} \\
\hline Attribute & $\begin{array}{c}\text { No. of selective } \\
\text { attribute } \\
\text { weightings } \\
\text { (out of 10) }\end{array}$ & Attribute & $\begin{array}{c}\text { No. of selective } \\
\text { attribute } \\
\text { weightings } \\
\text { (out of 10) }\end{array}$ \\
\hline Count of oxygen & 10 & Count of UU & 6 \\
\hline Count of UU & 7 & Count of CA & 5 \\
\hline Count of Uracil & 7 & Count of carbon & 5 \\
\hline Count of nitrogen & 6 & & \\
\hline
\end{tabular}

Ten algorithms (PCA, SVM, Relief, Uncertainty, Gini Index, Chi Squared, Deviation, Rule, Information Gain, and Information Gain Ratio) were used to determine the most important nucleotide attributes for the prediction of $\mathrm{HCV}$ subtypes $1 \mathrm{a}$ and $1 \mathrm{~b}$ responders from non-responders $(A)$ and responders from relapsers (B). Common nucleotide attributes used for genotypes $1 \mathrm{a}$ and $1 \mathrm{~b}$ have been bolded. A: adenine, T: thymine, C: cytosine, G: guanine.

Analysing subtypes $1 \mathrm{a}$ and $1 \mathrm{~b}$ responder vs. nonresponder sequences, Naive Bayes achieved the highest accuracy of $74.17 \%$ and $89.17 \%$, respectively. The highest accuracy for responder vs. relapser sequences were $82.50 \%$ when Bayes kernel was ran on Relief and 78.33\%, when Bayes kernel was ran on Chi Squared dataset (Table 2A and B). Full results from Bayesian algorithms are presented in Additional files 2 and 3.

\section{Neural networks}

Neural networks are computational models that are capable of machine learning and pattern recognition and have been used as predictive models.

Analyzing subtypes $1 \mathrm{a}$ and $1 \mathrm{~b}$ responder vs. nonresponder sequences, the Auto MLP showed the best performance with $76.67 \%$ and $85.00 \%$ accuracy, respectively, when it was ran on SVM dataset. By analyzing responder and relapser sequences, the best accuracy values were $79.17 \%$ and $78.33 \%$ for subtypes $1 \mathrm{a}$ and $1 \mathrm{~b}$, respectively (Table 2A and B). The full results of neural networks are presented in Additional files 2 and 3.

\section{Discussion and Conclusion}

In the absence of an effective vaccine, treatment of HCVinfected patients is the only means to combat the disease. Until recently, IFN/RBV combination therapy has been commonly used to treat $\mathrm{HCV}$ patients. The introduction of replicon systems [26,27] encoding enzymatically functional viral proteins such as NS3 and NS5B has led to the discovery of new anti-HCV drugs including the two NS3 inhibitors, boceprevir and telaprovir. However, the new drugs result in an increase in therapy response when they are combined with IFN and RBV [5,28]. Several reports indicated that viral and host factors influence the combination therapy outcome. Pascu et al. [15] analysed three $\mathrm{HCV}$ genotype $1 \mathrm{~b}$ genomes and identified a number of mutations in a specific region in HCV NS5A named interferon sensitivity determining region (ISDR) that predicted therapy response. Following this report, other research groups identified specific regions in other HCV proteins that predicted treatment outcome [7-9,14,19]. The current study aimed to analyse nucleotide sequences of $\mathrm{HCV}$ isolates from responders, non-responders, and relapsers aiming to generate decision trees for the prediction of treatment outcome. Recently, we have shown that the comparison of large numbers of sequences using mining techniques (such as decision tree) generated supervised and unsupervised models suitable for identification of novel proteins involved in the malignancy of breast cancer and lung cancer [29,30] and genetic markers for characterization of olive cultivars [20]. Using the same approach, we identified new genetic determinants that play important functional roles in the thermostable proteins [31], halostable proteins [32], and P1B-ATPase heavy metal transporters [21]. Furthermore, feature selection techniques and other learning methods such as bipartite learning graph and semi-supervised algorithms have already been used in drug-target interactions and the capability of these methods in predicting drugtarget datasets has been proven [33]. In the present study, we used the same strategy to evaluate correlation between HCV gene attributes at nucleotide levels with treatment response. Different attribute weighting systems used unique patterns to define the most important attributes for classification of data. To assess the genetic markers that affect the response to treatment, we analysed sequences from the patients that responded (more than 3.4 logs decline in viral load) or failed to respond (less than 1.4 log decline in viral load) to therapy at 28 days post-treatment. Analysing both subtypes 1a and 1b, some attribute weighting algorithms predicted 


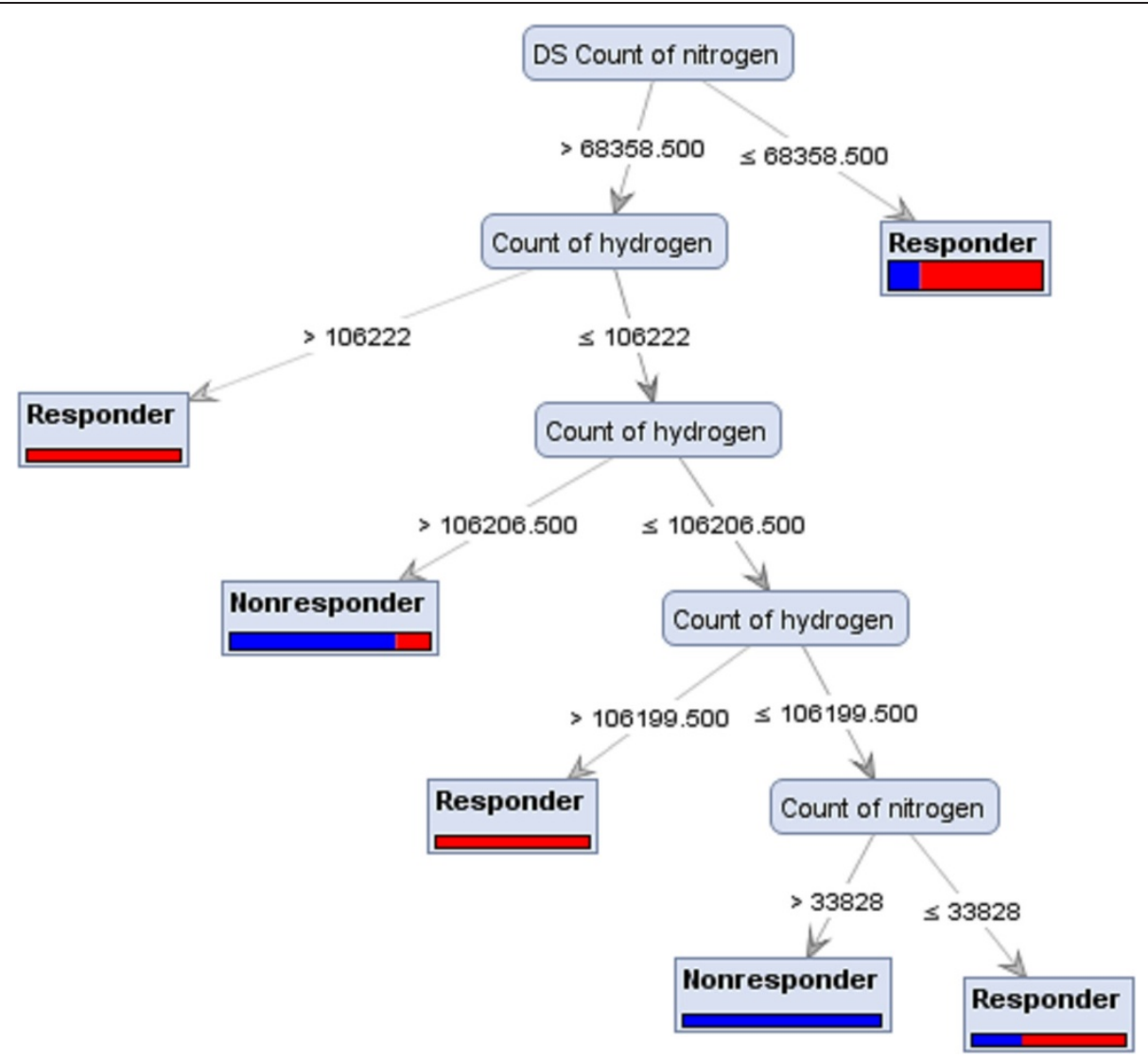

Figure 2 Achieved Decision Tree from Parallel model ran with Gini Index criterion on PCA dataset, which distinguish HCV subtype 1a responders' strains from non-responders strains.

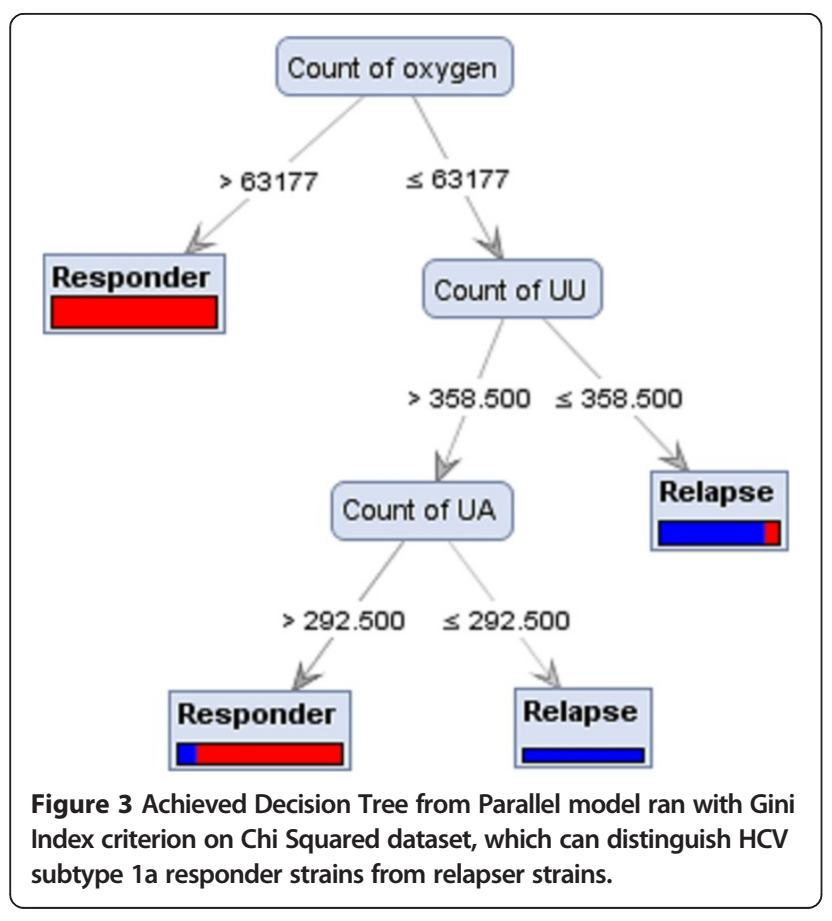

treatment response based on the UA count (Table 1A). The nucleotide composition of viral RNA may affect its sensitivity to IFN-stimulated genes (ISGs) such as RNase L which cleaves the RNA at dinucleotides UU and UA [34]. Interestingly, dinucleotide UU was selected by $60-70 \%$ models to distinguish responders from relapsers in both subtypes. Collectively, our analyses highlight the importance of dinucleotides UA and UU in combination treatment outcome. Strikingly, count of oxygen was an important attribute only when subtype 1a responders were compared to non-responders and relapsers (Table 1A and B). Our analysis identified several other dinucleotides that were selected as determining attributes for distinguishing responders from nonresponders and relapsers. These attributes may contribute to viral RNA structures that promote/prevent interactions with ISGs; however further investigation is required to determine their significance and precise role in $\mathrm{HCV}$ therapy response. The method employed in this study can be used to explore other viral features such as amino acids compositions, mRNA, miRNA and protein features in addition to nucleotide features. 


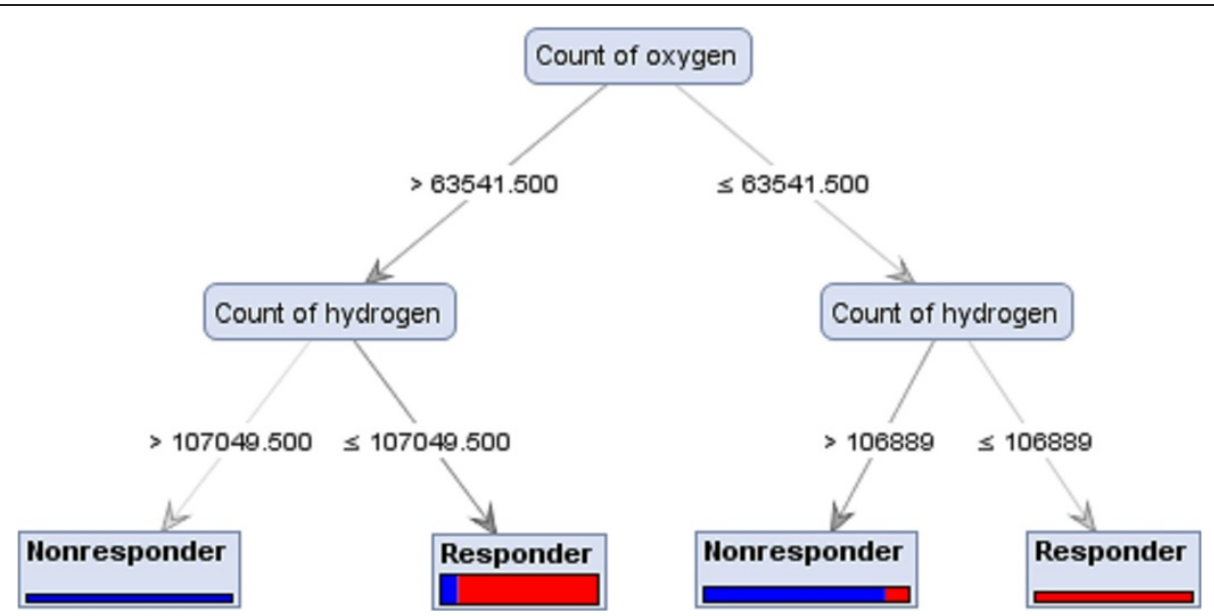

Figure 4 Achieved Decision Tree model ran with Gini Index criterion on PCA Dataset, which can distinguish HCV subtype 1b responder strains from non-responder strain.

Several mechanisms of action have been described for nucleotide analogue RBV but the exact mechanism are not fully understood [35]. Using an in vitro assay, Maag et al. [36] demonstrated that only high concentrations of RBV lead to its incorporation into HCV genome opposite of cytosine and uridine and terminated elongation process mediated by viral polymerase NS5B. Analysing subtypes $1 \mathrm{a}$ and $1 \mathrm{~b}$ responders and non-responders revealed that count of guanine was selected as an important attribute only in subtype 1a sequences. However, the accounts of guanine and adenine in viral genome are not

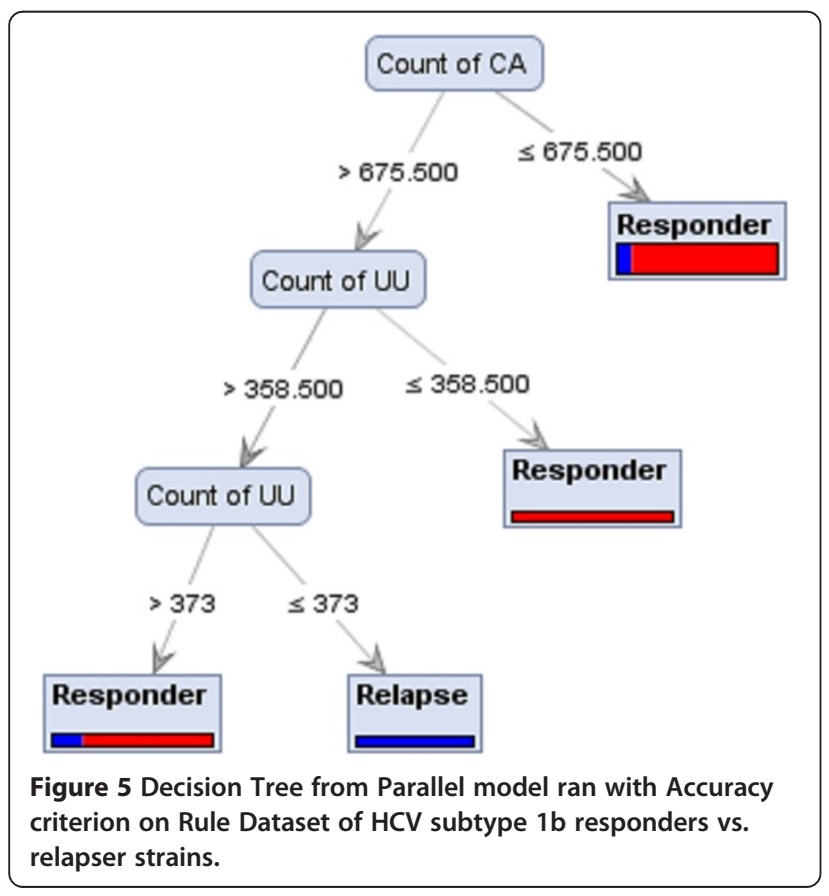

common selected determinant between both subtypes and do not support the proposed above mechanism of action of RBV at physiological concentrations. In line with our finding, it has been reported that using nucleotide analogues for treatment of HIV infections does not select for the nucleotide compositions of the viral genome [37].

The majority of induction tree models failed to distinguish between responders and relapsers and nonresponders. Decision Tree and Decision Tree parallel models generated decision trees with a reasonably high accuracy for the prediction of therapy response. Some tree induction models had simple configuration with three or four branches (Figure 4) however the depth of trees in some models were more complicated (Figure 2). The highest accuracies values for the prediction of subtypes $1 \mathrm{a}$ and $1 \mathrm{~b}$ responders from non-responders were $69.17 \%$ and $80.00 \%$, respectively. In addition, the sensitivity and specificity of these models were 0.88 and 0.60 for subtype $1 \mathrm{a}$ and 0.90 and 0.65 for subtype $1 \mathrm{~b}$. When responder and relapser groups were analysed the highest accuracy, $81.67 \%$, was achieved for both subtypes 1a and $1 \mathrm{~b}$ and the sensitivity and specificity of these models reached up to 0.80 and 0.85 for subtype $1 \mathrm{a}$ and 1.00 and 0.14 for subtype $1 \mathrm{~b}$, respectively (Table $2 \mathrm{~A}$ and $\mathrm{B}$ ).

The prediction performances of Neural Net algorithm in analyzing responders vs. non-responders sequences showed that AutoMLp algorithm could be used for prediction with accuracies up to 76.67 for subtype 1a and $85.00 \%$ for subtype 1 b. Also analysing subtype 1a responders vs. relapsers sequences revealed that Neural Network algorithm had the best prediction with accuracy up to 79.17 and sensitivity and specificity as high as 0.92 and 0.55 , respectively; while in analyzing subtype $1 \mathrm{~b}$ the Perceptron algorithm showed the highest accuracy 
Table 2 The highest values for accuracy, AUC, F-measure, precision, recall, sensitivity, and specificity for predicting responders vs. non-responders (A) and responders vs. relapsers (B) groups

\begin{tabular}{|c|c|c|c|c|c|c|c|c|}
\hline & \multicolumn{4}{|c|}{ Subtype 1a (Responders vs. Non-Responders) } & \multicolumn{4}{|c|}{ Subtype 1b (Responders vs. Non-Responders) } \\
\hline & Bayes & $\begin{array}{c}\text { Neural } \\
\text { Networks }\end{array}$ & SVM & Decision Trees & Bayes & $\begin{array}{c}\text { Neural } \\
\text { Networks }\end{array}$ & SVM & Decision Trees \\
\hline Database & Chi Squared & SVM & Relief & PCA & SVM & SVM & Relief & Gini Index \\
\hline Algorithm & $\begin{array}{l}\text { Naive Bayes } \\
\text { (Kernel) }\end{array}$ & AutoMLp & SVM & $\begin{array}{l}\text { DT Parallel Gini } \\
\text { Index }\end{array}$ & $\begin{array}{l}\text { Naive Bayes } \\
\text { (Kernel) }\end{array}$ & AutoMLp & SVM & $\begin{array}{l}\text { DT Random Forest Info } \\
\text { Gain }\end{array}$ \\
\hline Accuracy & $74.17 \%$ & $76.67 \%$ & $74.17 \%$ & $69.17 \%$ & $89.17 \%$ & $85.00 \%$ & $75.00 \%$ & $80.00 \%$ \\
\hline AUC & 0.84 & 0.68 & 0.75 & 0.59 & 0.94 & 0.94 & 0.84 & 0.83 \\
\hline $\begin{array}{c}\text { AUC } \\
\text { (optimistic) }\end{array}$ & 0.84 & 0.68 & 0.75 & 0.83 & 0.94 & 0.94 & 0.84 & 0.85 \\
\hline $\begin{array}{c}\text { AUC } \\
\text { (pessimistic) }\end{array}$ & 0.84 & 0.68 & 0.75 & 0.58 & 0.94 & 0.94 & 0.84 & 0.80 \\
\hline F-Measure & 0.78 & 0.82 & 0.80 & 0.73 & 0.92 & 0.87 & 0.80 & 0.86 \\
\hline Precision & 0.84 & 0.82 & 0.80 & 0.80 & 0.93 & 0.94 & 0.81 & 0.87 \\
\hline Recall & 0.73 & 0.82 & 0.88 & 0.73 & 0.93 & 0.83 & 0.83 & 0.90 \\
\hline Sensitivity & 0.73 & 0.82 & 0.88 & 0.73 & 0.93 & 0.83 & 0.83 & 0.90 \\
\hline Specificity & 0.85 & 0.75 & 0.60 & 0.65 & 0.85 & 0.80 & 0.50 & 0.65 \\
\hline \multicolumn{9}{|l|}{ B } \\
\hline & \multicolumn{4}{|c|}{ Subtype 1a (Responders vs. Relapsers) } & \multicolumn{4}{|c|}{ Subtype 1b (Responders vs. Relapsers) } \\
\hline & Bayes & $\begin{array}{c}\text { Neural } \\
\text { Networks }\end{array}$ & SVM & Decision Trees & Bayes & $\begin{array}{c}\text { Neural } \\
\text { Networks }\end{array}$ & SVM & Decision Trees \\
\hline Database & Chi Squared & SVM & Relief & PCA & SVM & SVM & Relief & Gini Index \\
\hline Algorithm & $\begin{array}{l}\text { Naive Bayes } \\
\text { (Kernel) }\end{array}$ & AutoMLp & SVM & $\begin{array}{l}\text { DT Parallel Gini } \\
\text { Index }\end{array}$ & $\begin{array}{l}\text { Naive Bayes } \\
\text { (Kernel) }\end{array}$ & AutoMLp & SVM & $\begin{array}{l}\text { DT Random Forest Info } \\
\text { Gain }\end{array}$ \\
\hline Accuracy & $82.50 \%$ & $79.17 \%$ & $82.50 \%$ & $81.67 \%$ & $78.33 \%$ & $78.33 \%$ & $84.17 \%$ & $81.67 \%$ \\
\hline AUC & 0.89 & 0.79 & 0.82 & 0.61 & & 0.00 & & 0.66 \\
\hline $\begin{array}{c}\text { AUC } \\
\text { (optimistic) }\end{array}$ & 0.89 & 0.79 & 0.82 & 0.91 & & 0.85 & & 0.85 \\
\hline $\begin{array}{c}\text { AUC } \\
\text { (pessimistic) }\end{array}$ & 0.89 & 0.79 & 0.82 & 0.74 & & 0.15 & & 0.47 \\
\hline F-Measure & 0.84 & 0.86 & 0.86 & 0.84 & 0.87 & 0.87 & 0.91 & 0.89 \\
\hline Precision & 0.92 & 0.83 & 0.90 & 0.90 & 0.78 & 0.78 & 0.84 & 0.82 \\
\hline Recall & 0.82 & 0.92 & 0.87 & 0.80 & 1.00 & 1.00 & 1.00 & 1.00 \\
\hline Sensitivity & 0.82 & 0.92 & 0.87 & 0.80 & 1.00 & 1.00 & 1.00 & 1.00 \\
\hline Specificity & 0.85 & 0.55 & 0.75 & 0.85 & 0.00 & 0.00 & 0.29 & 0.14 \\
\hline
\end{tabular}

with $78.33 \%$ and its sensitivity was 1.00 though its specificity was far low (Table 2A and B).

SVM is a supervised non-parametric statistical learning technique, therefore there is no assumption problems usually involve identification of multiple classes. From seven models of SVM applied on 11 datasets of subtypes 1a and $1 \mathrm{~b}$ in this study, the accuracies of SVMs algorithm were high $(74.14 \%$ for $1 \mathrm{a}$ and $75.00 \%$ for $1 \mathrm{~b})$ for responders vs. non-responders sequences in both subtypes. By comparing responders vs. relapsers sequences, SVM algorithm showed 82.50\% accuracy for subtype 1a while SVM LibSVM demonstrate the highest accuracy $(84.17 \%)$ for subtype $1 \mathrm{~b}$ (Table 2A and B).
When Naïve Bayes models trained with machine learning models, the performances of Naïve Bayes Kernel models were generally higher than models ran with Naïve Bayes (without Kernel). The highest possible accuracies gained when Naïve kernel Base models ran on the responders vs. non-responders sequences $(74.17 \%$ and $89.17 \%$ for subtypes $1 \mathrm{a}$ and $1 \mathrm{~b}$, respectively) and the sensitivity and specificity reached up to 0.73 and 0.85 for subtype $1 \mathrm{a}$, and 0.93 and 0.85 for subtype $1 \mathrm{~b}$. These achieved criteria make Naïve Bayes Kernel one of the best prediction models in comparing responders vs. non-responders sequences. Our findings suggested that Naïve-Based Kernel model has the best prediction accuracy in comparing 
responders vs. relapsers' sequences with figures up to $82.50 \%$ and $78.33 \%$ for subtypes $1 \mathrm{a}$ and $1 \mathrm{~b}$, respectively. Therefore, this model could be regarded as the most suitable algorithm for prediction responders vs. relapsers' sequences (Table $2 \mathrm{~A}$ and $\mathrm{B}$ ).

In conclusion, we applied combination of different algorithms on the genomic attributes of HCV subtypes 1a and $1 \mathrm{~b}$ and identified novel genetic markers including several dinucleotides that predicted therapy response rate with high accuracy.

\section{Methods}

Ninety three full length nucleotide sequences from subtypes 1a (12 relapsers, 22 responders and 13 nonresponders) and $1 \mathrm{~b}$ (7 relapsers, 26 responders and 13 non-responders) $[14,19]$ were extracted from GenBank. Further information regarding the outcome of the treatment was extracted from Virahep study (publically available) by Dr Donlin, Department of Biochemistry \& Molecular Biology, Saint Louis University School of Medicine, USA. Some original sequences contained deletions at their extreme ends. To avoid the effects of length factor of the sequences, the 93 sequences were aligned and adjusted by removing the extreme ends of all other sequences. The above sequences have been obtained by direct sequencing of PCR products from pre-treatment samples [19]. The adjusted nucleotide sequences of HCV subtypes $1 \mathrm{a}$ and $1 \mathrm{~b}$ were divided into 2 groups thereby creating four datasets; responders vs. non-responders, responders vs. relapsers for each subtype. Seventy six gene attributes - e.g. count and frequency of each nucleotide, di-nucleotides and elements, and molarities of salt contents (the concentration of monovalent cations in units of molar) were extracted using various bioinformatics tools and various software including CLC bio software (CLC bio, Finlandsgade 1012, Katrinebjerg 8200 Aarhus N Denmark). List of each attributes and calculated values are presented in Additional files 4 and 5 . Treatment types feature were categorical, and the other attributes were continuous variables. A dataset of these gene attributes was imported into Rapid Miner software [RapidMiner 5.0.001, Rapid-I GmbH, Stochumer Str. 475, 44227 Dortmund, Germany]. The null data for treatment type attribute was excluded, and this attribute was set as the output variable and the other variables were set as input variables.

\section{Data filtering}

We did not detect any duplicated attributes (two examples were assumed equal if all values of all selected attributes were equal) in our datasets. Related (with Pearson correlation greater than 0.9) and useless attributes were excluded from the dataset. Numerical attributes which possessed standard deviations less than or equal to a given deviation threshold (0.1) were considered useless. The final dataset was named final cleaned database (FCdb).

\section{Attribute weighting}

To identify the most important gene attributes, and to find likely patterns in ones that contribute to HCV therapy responses, 10 different algorithms of attribute weightings namely Information Gain, Information Gain Ratio, Rule, Deviation, Chi Squared, Gini Index, Uncertainty, Relief, Support Vector Machine (SVM) and PCA were applied to the FCbd as described previously [22,23,38].

Following attribute weighting application, each attribute gained a value between $0-1$ indicating the importance of it. All variables with weights equal to or higher than 0.5 selected and saved as new dataset; consequently, 10 new datasets were created. These newly formed datasets named according to their applied attribute weighting models (Information gain, Information gain ratio, Rule, Deviation, Chi Squared, Gini index, Uncertainty, Relief, SVM and PCA).

\section{Machine learning algorithms}

Three classes of machine learning algorithms namely Trees Inductions, SVM, and Bayesian along with the Neural Network were used to analyze the 11 datasets (see above) and construct models suitable for the prediction of $\mathrm{HCV}$ therapy response. To calculate the performance of each algorithm, 10-fold cross validation [39] was used to train and test models on all patterns. The classifier was trained on $90 \%$ of the data, and the remaining $10 \%$ were used as an unseen test set to assess the classifier's performance. This procedure was repeated 10 times (10-folds), with a different $10 \%$ of the data randomly selected as the test set in each repeat $[40,41]$. In this study, accuracy is calculated by taking the percentage of correct predictions over the total number of examples. Correct prediction means examples where value of prediction attribute is equal to the value of label attribute.

\section{Trees induction algorithms}

Four Trees Induction algorithms namely Decision Tree, Decision Tree Parallel, Decision Stump and Random Forest were run on the 11 datasets described in attribute weighting section. Each tree induction algorithms ran with Gain Ratio, Information Gain, and Gini Index and accuracy criteria. All algorithms except Random Forest created only one decision tree model; on the other hand Random Forest algorithm generated 10 different trees models for each criteria and consequently 572 trees were generated by all algorithms [38]. All models were trained and tested with 10 -fold cross validation and the averages of the accuracy values were calculated. 


\section{SVMs}

SVMs are theoretically well-established intuitive and feasible techniques for classification and prediction of supervised data [42-47]. We used seven SVMs models to classify and predict the HCV treatment response.

SVM learner uses the Java implementation of the support vector machine mySVM by Stefan Rueping [48]. This learning method can be used for both regression and classification and provides a fast algorithm and good results for many learning tasks. mySVM works with linear or quadratic and even asymmetric loss functions. This algorithm used dot kernel type for its learning process.

LIBSVM is an integrated software for support vector classification, (C-SVC, nu-SVC), regression (epsilon-SVR, nu-SVR) and distribution estimation (one-class SVM). It supports multi-class classification. The kernel type used for this algorithm was rbf.

Fast Large Margin learner is based on the linear support vector-learning scheme proposed by R.-E. Fan, K.-W. Chang, C.-J.Hsieh, X.-R.Wang, and C.-J. Lin. Although its results is similar to those delivered by classical SVM or logistic regression implementations, this linear classifier is able to work on data set with millions of examples and attributes.

Linear SVM is an extremely fast machine learning (data mining) algorithm for solving multi-class classification problems from ultra large data sets that implements an original proprietary version of a cutting plane algorithm for designing a linear SVM. Linear SVM is a linearly scalable routine meaning that it creates an SVM model in a CPU time, which scales linearly with the size of the training data set.

Evolutionary SVM (ESVMs) incorporate the learning engine of SVMs but develop the coefficients of the decision function by means of evolutionary algorithms. This algorithm used radial kernel type in this study.

SVMPSO is initialized with a group of random particles (solutions) and then searches for most efficient particles by updating each generation [49]. This algorithm trained with radial kernel type.

Hyper SVM model identifies the best parameter orders to reproduce a new classification. This model utilizes a Huffman-Tree like mechanism, called hyperSVM [50]. Briefly, main database (FCdb) transformed to SVM format and scaled by grid search (to avoid attributes in greater numeric ranges dominating those in smaller numeric ranges) and to find the optimal values for operator parameters. Datasets were divided into 10 subsets; 9 training subsets and 1 testing subset.

To ensure the best performance of SVM models, different parameters including accuracies, specificity, sensitivity, F-measure, and AUC (pessimist and optimist) were calculated.

\section{Naïve Bayes}

Naïve Bayes is based on the Bayes conditional probability Rule and represents an attractive classification tool when predictors are statistically independent. Two classification models namely Naïve base and Naïve base kernel trained with 10-fold cross validation on all 11 datasets to predict responders/relapsers and nonresponders. The accuracy of models was estimated as described [29].

\section{Neural network}

Two neural net algorithms (Neural Net and AutoMLP) trained with 10-fold cross validation on all 11 databases and the model accuracies in predicting the right protein's class computed as stated before.

Neural Net is a procedure that trains a neural net using a feed-forward neural network algorithm. A feedforward network is an artificial neural network with an algorithm where the connections between the modeled neurons are such that the information propagation is in one direction, from the input node, through intermediate nodes, to the output node.

A multilayer perceptron (MLP) is a feed-forward artificial neural network model that maps sets of input data on a set of suitable output. An MLP contains of multiple layers of nodes in a directed graph. Except for the input nodes, each node is a processing element that has a nonlinear activation function. MLP employs back propagation for training the network. This class of networks includes multiple layers of computational elements, usually interconnected in a feed-forward way. In many applications, the units of these networks apply a sigmoid function as an activation function.

In this study, a default-hidden layer with sigmoid type and size (number of attributes + number of classes) $/ 2+$ 1 created and added to the net and the training cycle was set to 500. The used activation function was the usual sigmoid function. Therefore, the values ranges of the attributes scaled to -1 and +1 . The type of the output node is sigmoid because the learning data described a classification task.

AutoMLP algorithm combines ideas from genetic algorithms as well as stochastic optimization [51]. The method maintains a small ensemble of networks that are trained in parallel with different rates and different numbers of hidden units. After a small, fixed number of epochs, the error rate is determined on a validation set and the worst performers are replaced with copies of the best networks, modified to have different numbers of hidden units and learning rates. Hidden unit numbers and learning rates are drawn according to probability distributions derived from successful rates and sizes. 


\section{Additional files}

\section{Additional file 1: The attribute weighting results of $1 \mathrm{a}$ and $1 \mathrm{~b}$ virus subtypes.}

Additional file 2: The prediction performances of $1 \mathrm{~b}$ virus subtype treatment groups.

Additional file 3: The prediction performances of 1a virus subtype treatment groups.

Additional file 4: List of calculated attributes for each sequence.

Additional file 5: The initial created datasets of $1 \mathrm{a}$ and $1 \mathrm{~b}$ virus subtypes.

\section{Competing interests}

The authors declare that they have no competing interests.

\section{Authors' contributions}

$\mathrm{GH}$ and ME identified the concept and designed the strategic plans of the experiments. AHK performed the data analysis experiments. AHK, GH and ME prepared the manuscript. All authors read and approved the final manuscript.

\section{Acknowledgements}

We would like to thank Dr. Maureen J. Donlin, Dept. of Biochemistry \& Molecular Biology, Saint Louis University School of Medicine, USA, for extraction of treatment outcome data from the Virahep study (publically available). This study supported by bioinformatics research group, University of Qom.

Received: 27 March 2014 Accepted: 10 August 2014

Published: 23 August 2014

\section{References}

1. Choo QL, Kuo G, Weiner AJ, Overby LR, Bradley DW, Houghton M: Isolation of a CDNA clone derived from a blood-borne non- $A$, non- $B$ viral hepatitis genome. Science 1989, 244(4902):359-362.

2. Shepard CW, Finelli L, Alter MJ: Global epidemiology of hepatitis C virus infection. Lancet Infect Dis 2005, 5(9):558-567.

3. Prentoe J, Jensen TB, Meuleman P, Serre SB, Scheel TK, Leroux-Roels G, Gottwein JM, Bukh J: Hypervariable region 1 differentially impacts viability of hepatitis $C$ virus strains of genotypes 1 to 6 and impairs virus neutralization. J Virol 2011, 85(5):2224-2234.

4. McHutchison JG, Poynard T: Combination therapy with interferon plus ribavirin for the initial treatment of chronic hepatitis C. Semin Liver Dis 1999, 19(Suppl 1):57-65.

5. Zeuzem S, Andreone P, Pol S, Lawitz E, Diago M, Roberts S, Focaccia R, Younossi Z, Foster GR, Horban A, Ferenci P, Nevens F, Mullhaupt B, Pockros P, Terg R, Shouval D, van Hoek B, Weiland O, Van Heeswijk R, De Meyer S, Luo D, Boogaerts G, Polo R, Picchio G, Beumont M: Telaprevir for retreatment of HCV infection. N Engl J Med 2011, 364(25):2417-2428.

6. Flamm SL, Lawitz E, Jacobson I, Bourlière M, Hezode C, Vierling JM, Bacon BR, Niederau C, Sherman M, Goteti V: Boceprevir with peginterferon alfa-2a-ribavirin is effective for previously treated chronic hepatitis $C$ genotype 1 infection. Clin Gastroenterol Hepatol 2013, 11(1):81-87. e84.

7. Akuta N, Suzuki F, Hirakawa M, Kawamura Y, Yatsuji H, Sezaki H, Suzuki Y, Hosaka T, Kobayashi M, Saitoh S, Arase Y, Ikeda K, Kumada H: Association of amino acid substitution pattern in core protein of hepatitis $C$ virus genotype 2a high viral load and virological response to interferonribavirin combination therapy. Intervirology 2009, 52(6):301-309.

8. Akuta N, Suzuki F, Sezaki H, Suzuki Y, Hosaka T, Someya T, Kobayashi M, Saitoh S, Watahiki S, Sato J, Matsuda M, Arase Y, Ikeda K, Kumada H: Association of amino acid substitution pattern in core protein of hepatitis $C$ virus genotype $1 \mathrm{~b}$ high viral load and non-virological response to interferon-ribavirin combination therapy. Intervirology 2005, 48(6):372-380

9. Lara J, Xia G, Purdy M, Khudyakov Y: Coevolution of the hepatitis C virus polyprotein sites in patients on combined pegylated interferon and ribavirin therapy. J Virol 2011, 85(7):3649-3663.

10. Hung $\mathrm{CH}$, Lee $\mathrm{CM}$, Lu SN, Lee JF, Wang JH, Tung HD, Chen TM, Hu TH, Chen WJ, Changchien CS: Mutations in the NS5A and E2-PePHD region of hepatitis $C$ virus type $1 \mathrm{~b}$ and correlation with the response to combination therapy with interferon and ribavirin. J Viral Hepat 2003, 10(2):87-94.
11. de Munoz Rueda P, Casado J, Paton R, Quintero D, Palacios A, Gila A, Quiles R, Leon J, Ruiz-Extremera A, Salmeron J: Mutations in E2-PePHD, NS5A-PKRBD, NS5A-ISDR, and NS5A-V3 of hepatitis C virus genotype 1 and their relationships to pegylated interferon-ribavirin treatment responses. J Virol 2008, 82(13):6644-6653.

12. Yang SS, Lai MY, Chen DS, Chen GH, Kao JH: Mutations in the NS5A and E2PePHD regions of hepatitis $C$ virus genotype $1 b$ and response to combination therapy of interferon plus ribavirin. Liver Int 2003, 23(6):426-433.

13. Kadokura M, Maekawa S, Sueki R, Miura M, Komase K, Shindo H, Amemiya F, Uetake T, Inoue T, Sakamoto M, Nakagawa M, Sakamoto N, Watanabe M, Enomoto N: Analysis of the complete open reading frame of genotype $2 \mathrm{~b}$ hepatitis $\mathrm{C}$ virus in association with the response to peginterferon and ribavirin therapy. PLoS One 2011, 6:e24514.

14. Cannon NA, Donlin MJ, Fan X, Aurora R, Tavis JE: Hepatitis C virus diversity and evolution in the full open-reading frame during antiviral therapy. Plos One 2008, 3(5):e2123.

15. Pascu M, Martus P, Hohne M, Wiedenmann B, Hopf U, Schreier E, Berg T: Sustained virological response in hepatitis $C$ virus type $1 \mathrm{~b}$ infected patients is predicted by the number of mutations within the NS5A-ISDR: a meta-analysis focused on geographical differences. Gut 2004, 53(9):1345-1351.

16. Hamano K, Sakamoto N, Enomoto N, Izumi N, Asahina Y, Kurosaki M, Ueda E, Tanabe Y, Maekawa S, Itakura J, Watanabe H, Kakinuma S, Watanabe M: Mutations in the NS5B region of the hepatitis $C$ virus genome correlate with clinical outcomes of interferon-alpha plus ribavirin combination therapy. J Gastroenterol Hepatol 2005, 20:1401-1409.

17. Ge D, Fellay J, Thompson AJ, Simon JS, Shianna KV, Urban TJ, Heinzen EL, Qiu P, Bertelsen AH, Muir AJ, Sulkowski M, McHutchison JG, Goldstein DB: Genetic variation in IL28B predicts hepatitis C treatment-induced viral clearance. Nature 2009, 461:399-401.

18. Suppiah V, Moldovan M, Ahlenstiel G, Berg T, Weltman M, Abate ML, Bassendine M, Spengler U, Dore GJ, Powell E, Riordan S, Sheridan D, Smedile A, Fragomeli V, Muller T, Bahlo M, Stewart GJ, Booth DR, George J: $\mathrm{IL} 28 \mathrm{~B}$ is associated with response to chronic hepatitis $C$ interferon-alpha and ribavirin therapy. Nat Genet 2009, 41:1100-1104.

19. Donlin MJ, Cannon NA, Yao E, Li J, Wahed A, Taylor MW, Belle SH, Di Bisceglie AM, Aurora R, Tavis JE: Pretreatment sequence diversity differences in the full-length hepatitis $C$ virus open reading frame correlate with early response to therapy. J Virol 2007, 81(15):8211-8224.

20. Beiki AH, Saboor S, Ebrahimi M: A new avenue for classification and prediction of olive cultivars using supervised and unsupervised algorithms. PLoS One 2012, 7(9):e44164.

21. Ashrafi E, Alemzadeh A, Ebrahimi M, Ebrahimie E, Dadkhodaei N, Ebrahimi M: Amino Acid Features of P1B-ATPase Heavy Metal Transporters Enabling Small Numbers of Organisms to Cope with Heavy Metal Pollution. Bioinform Biol Insights 2011, 5:59-82.

22. Ebrahimie E, Ebrahimi M, Sarvestani NR: Protein attributes contribute to halo-stability, bioinformatics approach. Saline Systems 2011, 7(1):1.

23. Ebrahimi M, Lakizadeh A, Agha-Golzadeh P, Ebrahimie E: Prediction of thermostability from amino acid attributes by combination of clustering with attribute weighting: a new vista in engineering enzymes. PLOS One 2011, 6(8):e23146.

24. Gromiha MM: Prediction of protein stability upon point mutations. Biochem Soc Trans 2007, 35(Pt 6):1569-1573.

25. Mitra S, Acharya T: Data Mining: Multimedia. Soft Computing, and Bioinformatics Wiley, New York; 2003.

26. Lohmann V, Korner F, Koch J, Herian U, Theilmann L, Bartenschlager R: Replication of subgenomic hepatitis $C$ virus RNAs in a hepatoma cell line. Science 1999, 285(5424):110-113.

27. Blight KJ, Kolykhalov AA, Rice CM: Efficient initiation of HCV RNA replication in cell culture. Science 2000, 290(5498):1972-1974.

28. Bacon BR, Gordon SC, Lawitz E, Marcellin P, Vierling JM, Zeuzem S, Poordad F, Goodman ZD, Sings HL, Boparai N, Burroughs M, Brass CA, Albrecht JK, Esteban R: Boceprevir for previously treated chronic HCV genotype 1 infection. N Engl J Med 2011, 364:1207-1217.

29. Ebrahimi M, Ebrahimie E, Shamabadi N, Ebrahimi M: Are there any differences between features of proteins expressed in malignant and benign breast cancers? J Res Med Sci 2010, 15(6):299-309.

30. Hosseinzadeh F, Kayvanjoo AH, Ebrahimi M, Goliaei B: Prediction of lung tumor types based on protein attributes by machine learning algorithms. Springerplus 2013, 2(1):238. 
31. Ebrahimi M, Ebrahimie E: Sequence-based prediction of enzyme thermostability through bioinformatics algorithms. Curr Bioinformatics 2010, 5:195-203.

32. Ebrahimie E, Ebrahimi M, Rahpayma M: Investigating protein features contribute to salt stability of halolysin proteins. J Cell Mol Res 2010, 2(1):15-28.

33. Yu W, Jiang Z, Wang J, Tao R: Using feature selection technique for drugtarget interaction networks prediction. Curr Med Chem 2011, 18(36):5687-5693.

34. Player MR, Torrence PF: The 2-5A system: modulation of viral and cellular processes through acceleration of RNA degradation. Pharmacol Ther 1998, 78:55-113.

35. Feld JJ, Hoofnagle $\mathrm{JH}$ : Mechanism of action of interferon and ribavirin in treatment of hepatitis C. Nature 2005, 436:967-972.

36. Maag D, Castro C, Hong Z, Cameron CE: Hepatitis C virus RNA-dependent RNA polymerase (NS5B) as a mediator of the antiviral activity of ribavirin. J Biol Chem 2001, 276:46094-46098.

37. van der Kuyl AC, Berkhout B: The biased nucleotide composition of the HIV genome: a constant factor in a highly variable virus. Retrovirology 2012, 9(1):92.

38. Hosseinzadeh F, Ebrahimi M, Goliaei B, Shamabadi N: Classification of Lung Cancer Tumors Based on Structural and Physicochemical Properties of Proteins by Bioinformatics Models. PLoS One 2012, 7(7):e40017.

39. Habashy HO, Powe DG, Glaab E, Ball G, Spiteri I, Krasnogor N, Garibaldi JM, Rakha EA, Green AR, Caldas C: RERG (Ras-like, oestrogen-regulated, growth-inhibitor) expression in breast cancer: a marker of ER-positive luminal-like subtype. Breast Cancer Res Treat 2011, 128(2):315-326.

40. Nowotny T, Rospars JP, Martinez D, Elbanna S, Anton S: Machine learning for automatic prediction of the quality of electrophysiological recordings. PLoS One 2013, 8(12):e80838.

41. Weiss SM, Kulikowski CA: Computer systems that learn: classification and prediction methods from statistics, neural nets, machine learning, and expert systems. San Mateo, CA: Morgan Kaufmann; 1991.

42. Radman A, Gredicak M, Kopriva I, Jeric I: Predicting antitumor activity of peptides by consensus of regression models trained on a small data sample. Int J Mol Sci 2011, 12(12):8415-8430.

43. Tan T, Platel B, Huisman H, Sánchez Cl, Mus R, Karssemeijer N: Computer-aided lesion diagnosis in automated 3-D breast ultrasound using coronal spiculation. IEEE Trans Med Imaging 2012, 31(5):1034-1042

44. Li Z, Liu P, Wang W, Xu C: Using support vector machine models for crash injury severity analysis. Accid Anal Prev 2012, 45(2):478-486.

45. Wang K, Hu X, Wang Z, Yan A: Classification of acetylcholinesterase inhibitors and decoys by a support vector machine. Comb Chem High Throughput Screen 2012, 15(6):492-502.

46. Kuo CC, Lin WS, Dressel CA, Chiu AW: Classification of intended motor movement using surface EEG ensemble empirical mode decomposition. Conf Proc IEEE Eng Med Biol Soc 2011, 2011:6281-6284.

47. Sawagashira T, Hayashi T, Hara T, Katsumata A, Muramatsu C, Zhou X, lida Y, Katagi K, Fujita H: An automatic detection method for carotid artery calcifications using top-hat filter on dental panoramic radiographs. Conf Proc IEEE Eng Med Biol Soc 2011, 2011:6208-6211.

48. Rüping S: mySVM-Manual. Dortmund: Computer Science Department, Al Unit, University of Dortmund; 2000.

49. Ardjani F, Sadouni K: Optimization of SVM Multiclass by Particle Swarm (PSO-SVM). IJMECS 2010, 2(2):32.

50. Shyu F-M, Liao H-Y: A hyper SVM model for multiple classifications. In Natural Computation (ICNC), 2011 Seventh International Conference on: 2011. Shanghai, China: IEEE; 340-343.

51. Breuel T, Shafait F: Automlp: Simple, effective, fully automated learning rate and size adjustment. In The Learning Workshop. Utah, USA: 2010.

Cite this article as: KayvanJoo et al:: Prediction of hepatitis C virus interferon/ribavirin therapy outcome based on viral nucleotide attributes using machine learning algorithms. BMC Research Notes 2014 7:565.

\section{Submit your next manuscript to BioMed Central and take full advantage of:}

- Convenient online submission

- Thorough peer review

- No space constraints or color figure charges

- Immediate publication on acceptance

- Inclusion in PubMed, CAS, Scopus and Google Scholar

- Research which is freely available for redistribution

Submit your manuscript at www.biomedcentral.com/submit
C Biomed Central 